\title{
Abrupt transition in the structural formation of
}

\section{interconnected networks}

\author{
Filippo Radicchi ${ }^{1,2 \star}$ and Alex Arenas ${ }^{3,4}$
}

Our world is linked by a complex mesh of networks through which information, people and goods flow. These networks are interdependent on each other, and present structural and dynamical features ${ }^{1-6}$ different from those observed in isolated networks ${ }^{7-9}$. Although examples of such dissimilar properties are becoming more abundant-such as in diffusion, robustness and competition-it is not yet clear where these differences are rooted. Here we show that the process of building independent networks into an interconnected network of networks undergoes a structurally sharp transition as the interconnections are formed. Depending on the relative importance of inter- and intra- layer connections, we find that the entire interdependent system can be tuned between two regimes: in one regime, the various layers are structurally decoupled and they act as independent entities; in the other regime, network layers are indistinguishable and the whole system behaves as a singlelevel network. We analytically show that the transition between the two regimes is discontinuous even for finite-size networks. Thus, any real-world interconnected system is potentially at risk of abrupt changes in its structure, which may manifest new dynamical properties.

Interacting, interdependent or multiplex networks are different ways of naming the same class of complex systems where networks are not considered as isolated entities but interacting with each other. In multiplex, the nodes at each network are instances of the same entity; thus, the networks are representing simply different categorical relationships between entities, and usually categories are represented by layers. Interdependent networks is a more general framework where nodes can be different at each network.

Many, if not all, real networks are coupled with other real networks. Examples can be found in several domains: social networks (for example, Facebook, Twitter and so on) are coupled because they share the same actors ${ }^{10}$; multimodal transportation networks are composed of different layers (for example, bus, subway and so on) that share the same locations ${ }^{11}$; the functioning of communication and power grid systems depends one on the other ${ }^{1}$. So far, all phenomena that have been studied on interdependent networks, including percolation ${ }^{1,3}$, epidemics ${ }^{4}$ and linear dynamical systems ${ }^{5}$, have provided results that differ much from those valid in the case of isolated complex networks. Sometimes the difference is radical: for example, whereas isolated scale-free networks are robust against failures of their nodes or edges ${ }^{12}$, scale-free interdependent networks are instead very fragile ${ }^{1,3}$.

Given such observations, two fundamentally important theoretical questions are in order: Why do dynamical and critical phenomena running on interdependent network models differ
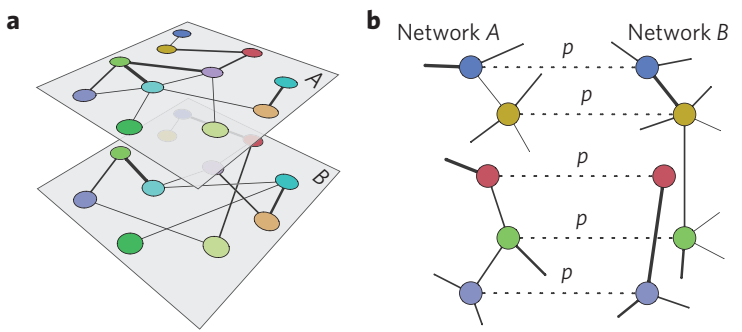

Figure 1 | Interdependent networks. a, Schematic example of two interdependent networks $A$ and $B$. In this representation, nodes of the same colour are one-to-one interdependent. $\mathbf{b}$, In our model, inter-layer edges have weights equal to $p$.

so much from their analogues in isolated networks? What are the regimes of applicability of the theory valid for isolated networks to interdependent networks? Here, we provide an analytic answer to both of these questions by characterizing the structural properties of the whole interconnected network in terms of the networks that compose it.

For simplicity, we consider here the case of two interdependent networks. The following method can be, however, generalized to an arbitrary number of interdependent networks and its solution is reported in the Supplementary Information. We assume that the two interdependent networks $A$ and $B$ are undirected and weighted, and that they have the same number of nodes $N$. The weighted adjacency matrices of the two graphs are indicated as $A$ and $B$, respectively, and they both have dimensions $N \times N$. With this notation, the element $A_{i j}=A_{j i}$ is equal to the weight of the connection between the nodes $i$ and $j$ in network $A$. The definition of $B$ is analogous.

We consider the case of one-to-one symmetric interdependency ${ }^{1}$ between nodes in the networks $A$ and $B$ (Fig. 1a). In the more general case of multiple interdependencies, the solution is qualitatively similar (see Supplementary Information). The connections between interdependent nodes of the two networks are weighted by a factor $p$ (see Fig. 1b), any other weighted factor for the networks $A$ and $B$ is implicitly absorbed in their weights. The supra-adjacency matrix $G$ of the whole network is therefore given by

$$
G=\left(\begin{array}{cc}
A & p \mathbb{1} \\
p \mathbb{1} & B
\end{array}\right)
$$

where $\mathbb{1}$ is the identity matrix of dimensions $N \times N$.

\footnotetext{
${ }^{1}$ Center for Complex Networks and Systems Research, School of Informatics and Computing, Indiana University, 47408 Bloomington, Indiana, USA,

${ }^{2}$ Departament d'Enginyeria Quimica, Universitat Rovira i Virgili, 43007 Tarragona, Spain, ${ }^{3}$ Departament d'Enginyeria Informàtica i Matemàtiques,

Universitat Rovira i Virgili, 43007 Tarragona, Spain, ${ }^{4}$ IPHES, Institut Catala de Paleoecologia Humana i Evoluci'o Social, 43007 Tarragona, Spain. 
Using this notation we can define the supra-Laplacian of the interconnected network as

$$
\mathcal{L}=\left(\begin{array}{cc}
\mathcal{L}_{A}+p \mathbb{1} & -p \mathbb{1} \\
-p \mathbb{1} & \mathcal{L}_{B}+p \mathbb{1}
\end{array}\right)
$$

The blocks present in $\mathcal{L}$ are square symmetric matrices of dimensions $N \times N$, In particular, $\mathcal{L}_{A}$ and $\mathcal{L}_{B}$ are the Laplacians of the networks $A$ and $B$, respectively.

Our investigation focuses on the analysis of the spectrum of the supra-Laplacian to ascertain the origin of the structural changes of the merging of networks in an interconnected system. The spectrum of the Laplacian of a graph is a fundamental mathematical object for the study of the structural properties of the graph itself. There are many applications and results on graph Laplacian eigenpairs and their relations to numerous graph invariants (including connectivity, expanding properties, genus, diameter, mean distance and chromatic number) as well as to partition problems (graph bisection, connectivity and separation, isoperimetric numbers, maximum cut, clustering, graph partition), and approximations for optimization problems on graphs (cutwidth, bandwidth, min-p-sum problems, ranking, scaling, quadratic assignment problem $)^{13-16}$.

Note that, for any graph, all eigenvalues of its Laplacian are non-negative numbers. The smallest eigenvalue is always equal to zero and the eigenvector associated with it is trivially a vector whose entries are all identical. The second smallest eigenvalue $\lambda_{2}$ also called the algebraic connectivity ${ }^{17}$ is one of the most significant eigenvalues of the Laplacian. It is strictly larger than zero only if the graph is connected. More importantly, the eigenvector associated with $\lambda_{2}$, which is called the characteristic valuation or Fiedler vector of a graph, provides even deeper information about its structure ${ }^{18-20}$. For example, the components of this vector associated with the various nodes of the network are used in spectral clustering algorithms for the bisection of graphs ${ }^{21}$.

Our approach consists of the study of the behaviour of the second smallest eigenvalue of the supra-Laplacian matrix $\mathcal{L}$ and its characteristic valuation as a function of $p$, given the single-layer network Laplacians $\mathcal{L}_{A}$ and $\mathcal{L}_{B}$.

According to the theorem of refs 22,23 (that is, the so-called min-max principle), the second smallest eigenvalue of $\mathcal{L}$ is given by

$$
\lambda_{2}(\mathcal{L})=\min _{|v\rangle \in \mathcal{V}}\langle v|\mathcal{L}| v\rangle
$$

where $|v\rangle \in \mathcal{V}$ is such that $\langle v \mid 1\rangle=0,\langle v \mid v\rangle=1$

The vector $|1\rangle$ has $2 N$ entries all equal to 1 . Equation (3) means that $\lambda_{2}(\mathcal{L})$ is equal to the minimum of the function $\langle v|\mathcal{L}| v\rangle$, over all possible vectors $|v\rangle$ that are orthogonal to the vector $|1\rangle$ and that have norm equal to one. The vector for which this minimum is reached is thus the characteristic valuation of the supra-Laplacian (that is, $\mathcal{L}|v\rangle=\lambda_{2}|v\rangle$ ).

We distinguish two blocks of size $N$ in the vector $|v\rangle$ by writing it as $|v\rangle=\left|v_{A}, v_{B}\right\rangle$. In this notation, $\left|v_{A}\right\rangle$ is the part of the eigenvector whose components correspond to the nodes of network $A$, and $\left|v_{B}\right\rangle$ is the part of the eigenvector whose components correspond to the nodes of network $B$. We can now write

$$
\begin{aligned}
\langle v|\mathcal{L}| v\rangle= & \left\langle v_{A}, v_{B}|\mathcal{L}| v_{A}, v_{B}\right\rangle=\left\langle v_{A}\left|\mathcal{L}_{A}\right| v_{A}\right\rangle+\left\langle v_{B}\left|\mathcal{L}_{B}\right| v_{B}\right\rangle \\
& +p\left(\left\langle v_{A} \mid v_{A}\right\rangle+\left\langle v_{B} \mid v_{B}\right\rangle-2\left\langle v_{A} \mid v_{B}\right\rangle\right)
\end{aligned}
$$

and the previous set of constraints as $\left\langle v_{A} \mid 1\right\rangle+\left\langle v_{B} \mid 1\right\rangle=0$ and $\left\langle v_{A} \mid v_{A}\right\rangle+\left\langle v_{B} \mid v_{B}\right\rangle=1$, where now all vectors have dimension $N$. Accounting for such constraints, we can finally rewrite the minimization problem as

$$
\lambda_{2}(\mathcal{L})=p+\min _{|v\rangle \in \mathcal{V}}\left\{\left\langle v_{A}\left|\mathcal{L}_{A}\right| v_{A}\right\rangle+\left\langle v_{B}\left|\mathcal{L}_{B}\right| v_{B}\right\rangle-2 p\left\langle v_{A} \mid v_{B}\right\rangle\right\}
$$

This minimization problem can be solved using Lagrange multipliers (see Supplementary Information for technical details).

In this way we are able to find that the second smallest eigenvalue of the supra-Laplacian matrix $\mathcal{L}$ is given by

$$
\lambda_{2}(\mathcal{L})= \begin{cases}2 p & , \text { if } p \leq p^{*} \\ \leq \frac{1}{2} \lambda_{2}\left(\mathcal{L}_{A}+\mathcal{L}_{B}\right) & , \text { if } p \geq p^{*}\end{cases}
$$

This indicates that the algebraic connectivity of the interconnected system follows two distinct regimes, one in which its value is independent of the structure of the two layers, and the other in which its upper bound is limited by the algebraic connectivity of the weighted superposition of the two layers whose Laplacian is given by $(1 / 2)\left(\mathcal{L}_{A}+\mathcal{L}_{B}\right)$. More importantly, the discontinuity in the first derivative of $\lambda_{2}$ is reflected in a radical change of the structural properties of the system happening at $p^{*}$ (see Supplementary Information). This pronounced change is visible in the coordinates of characteristic valuation of the nodes of the two network layers. In the regime $p \leq p^{*}$, the components of the eigenvector are

$$
\left|v_{A}\right\rangle=-\left|v_{B}\right\rangle \quad \text { where }\left|v_{A}\right\rangle= \pm \frac{1}{\sqrt{2 N}}|1\rangle
$$

This means that the two network layers are structurally disconnected and independent. For $p \geq p^{*}$, we have

$$
\left\langle v_{A} \mid 1\right\rangle=\left\langle v_{B} \mid 1\right\rangle=0
$$

which means that the components of the vector corresponding to interdependent nodes of network $A$ and $B$ have the same sign, whereas nodes in the same layer have alternating signs. Thus, in this second regime, the system connectivity is dominated by inter-layer connections, and the two network layers are structurally indistinguishable.

The critical value $p^{*}$ at which the transition occurs is the point at which we observe the crossing between the two different behaviours of $\lambda_{2}$, which means

$$
p^{*} \leq \frac{1}{4} \lambda_{2}\left(\mathcal{L}_{A}+\mathcal{L}_{B}\right)
$$

This upper bound becomes exact in the case of identical network layers (see Supplementary Information). As inter-layer connections have weights that grow with $p$, the transition happens at the point at which the weight of the inter-layer connections exceeds the half part of the inverse of the algebraic connectivity of the weighted super-position of both network layers (Fig. 2). In the case of $\ell$ network layers, the result is equivalent to the superposition of all of them (see Supplementary Information).

It is important to note that the discontinuity in the first derivative of $\lambda_{2}(\mathcal{L})$ can be interpreted as the consequence of the crossing of two different populations of eigenvalues (see the case of identical layers in the Supplementary Information). The same crossing will also happen for the other eigenpairs of the graph Laplacian (except for the smallest and the largest ones), and thus will reflect in the discontinuities in the first derivatives of the corresponding eigenvalues.

A physical interpretation of the algebraic phase transition that we are able to analytically predict can be given by viewing the function $\langle v|\mathcal{L}| v\rangle$ as an energy-like function. From this point of view, equation (3) becomes equivalent to a search for the ground-state energy, and the characteristic valuation can be viewed as the ground-state configuration. This analogy is straightforward if one realizes that equation (3) is equivalent to the minimization of the weighted cut of the entire networked system (whose adjacency matrix $G$ is defined in equation (1)), and that the minimum of this function corresponds to the ground state of a wide class of 

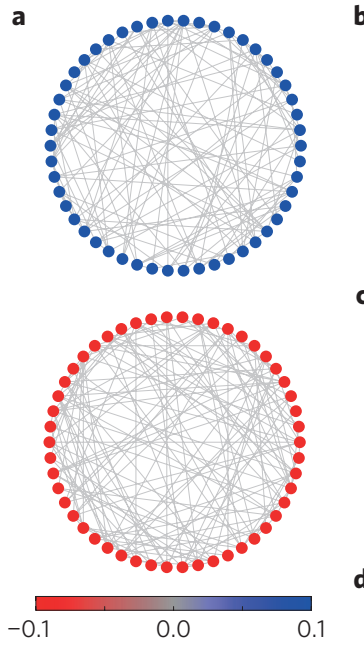

Characteristic valuation
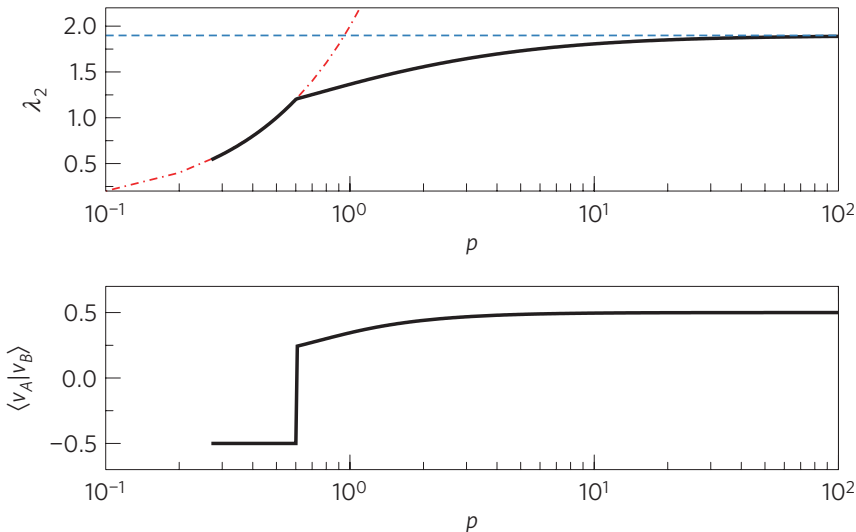

d

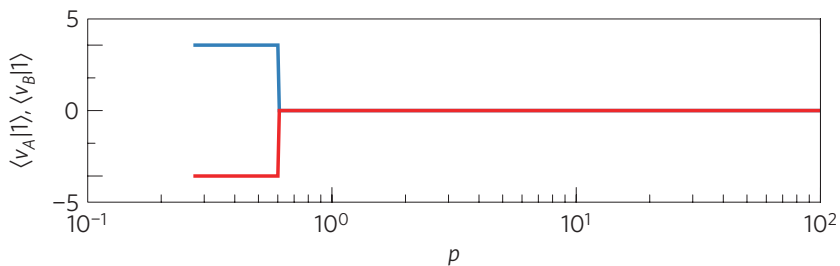

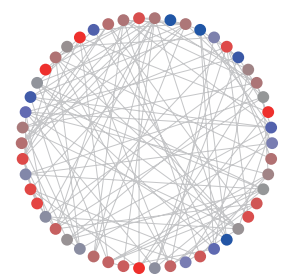

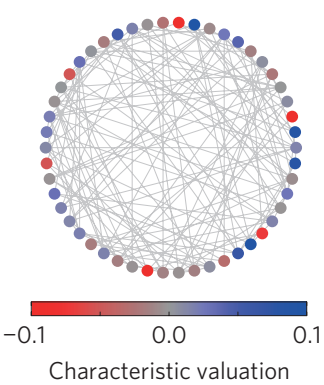

Characteristic valuation

Figure 2 | First-order structural transition in interdependent networks. Algebraic connectivity and Fiedler vector for two interdependent Erdös-Renyí networks of $N=50$ nodes and average degree $\bar{k}=5$. We consider a single realization of this model in which the critical point is $p^{*}=0.602(1)$.

$\mathbf{a}$, Characteristic valuation of the nodes in the two network layers for $p=0.602$. b. Algebraic connectivity of the system (black line). The discontinuity of the first derivative of $\lambda_{2}$ is very clear. The two different regimes $2 p$ and $\lambda_{2}\left(\mathcal{L}_{A}+\mathcal{L}_{B}\right) / 2$ are shown as red dot-dashed and blue dashed lines, respectively. c, Inner product $\left\langle v_{A} \mid v_{B}\right\rangle$ between the part of the Fiedler eigenvector $\left(\left|v_{A}\right\rangle\right)$ corresponding to nodes in the network $A$ and the one $\left(\left|v_{B}\right\rangle\right)$ corresponding to vertices in network $B$ as a function of $p$. $\mathbf{d}$, Inner products $\left\langle v_{A} \mid 1\right\rangle$ and $\left\langle v_{B} \mid 1\right\rangle$ as functions of $p .\left\langle v_{A} \mid 1\right\rangle$ and $\left\langle v_{B} \mid 1\right\rangle$ indicate the sum of all components of the Fiedler vectors $\left|v_{A}\right\rangle$ and $\left|v_{B}\right\rangle$, respectively. e, Characteristic valuation of the nodes in the two network layers for $p=0.603$.

energy functions ${ }^{24}$ and fitness landscapes ${ }^{25}$. These include, among others, the energy associated with the Ising spin models ${ }^{26}$ and cost functions of combinatorial optimization problems, such as the travelling salesman problem ${ }^{27}$. In summary, the structural transition of interdependent networks involves a discontinuity in the first derivative of an energy-like function, and thus, according to the Ehrenfest classification of phase transitions, it is a discontinuous transition ${ }^{28}$.

As the transition at the algebraic level has the same nature as the connectivity transition studied in ref. 1 in the same class of networked systems, it is worth discussing the relations between the two phase transitions. We can reduce our model to the annealed version of the model considered in ref. 1 by setting $A=t^{2} A, B=t^{2} B$ and $p=t$, with $1-t$ being the probability that one node in one of the networks fails (see Supplementary Information). All of the results stated so far hold, with only two different interpretations. First, the upper bound of equation (8) becomes a lower bound for the critical threshold of the algebraic transition that reads in terms of occupation probability as

$$
t_{c} \geq \frac{4}{\lambda_{2}\left(\mathcal{L}_{A}+\mathcal{L}_{B}\right)}
$$

Second, the way to look at the transition must be reversed: network layers are structurally independent (that is, the analogue of the non-percolating phase) for values of $t \leq t_{c}$, whereas they become algebraically connected (that is, the analogue of the percolating phase) when $t \geq t_{c}$.

As is well known, the algebraic connectivity represents a lower bound for both the edge connectivity and node connectivity of a graph (that is, respectively the minimal number of edges or nodes that should be removed to disconnect the graph $)^{17}$. Indeed, the algebraic connectivity of a graph is often used as a control parameter to make the graph more resilient to random failures of its nodes or edges ${ }^{29}$. Thus, the lower bound of equation (9) represents also a lower bound for the critical percolation threshold measured in ref. 1. Interestingly, our prediction turns out to be a sharp estimate of the lower bound. For the Erdős-Rényi model, we have in fact $t_{c} \geq 2 / \bar{k}$, if the two networks have the same average degree $\bar{k}$, and this value must be compared with $2.455 / \bar{k}$ as predicted in refs 1,3 . Similarly, we are able to predict that $t_{c}$ grows as the degree distribution of the network becomes more broad ${ }^{14}$, in the same way as numerically observed in ref. 1 .

Although we are not able to directly map the algebraic transition to the percolation one, we believe that the existence of a firstorder transition at the algebraic level represents indirect support for the discontinuity of the percolation transition. We further emphasize that the transition is effectively present only if $t_{c} \leq 1$, and thus according to equation (9) only if $\lambda_{2}\left(\mathcal{L}_{A}+\mathcal{L}_{B}\right) \geq 4$. This condition is verified for network layers that have a sufficiently large connectivity, and this qualitatively confirms the observation in ref. 30 regarding a change in the nature of the percolation phase transition in interdependent networks with variable numbers of interdependent nodes.

In conclusion, we would like to briefly discuss the deep practical implications of our results. The abrupt nature of the structural transition is, in fact, not only visible in the limit of infinitely large systems, but for networks of any size. Thus, even real networked systems composed of few elements may be subjected to abrupt structural changes, including failures. Our theory provides, however, fundamental aids for the prevention of such collapses. It allows, in fact, not only the prediction of the critical point of the transition, but, more importantly, to accurately design the structure of such systems to make them more robust. For example, the percolation threshold of interconnected systems can be simply decreased by increasing the algebraic connectivity of the superposition of the network layers. This means that an effective strategy to make an interdependent system more 
robust is to avoid the repetition of edges among layers, and thus bring the superposition of the layers as close as possible to an all-to-all topology.

Received 18 April 2013; accepted 15 August 2013; published online 22 September 2013

\section{References}

1. Buldyrev, S. V. et al. Catastrophic cascade of failures in interdependent networks. Nature 464, 1025-1028 (2010).

2. Gao, J., Buldyrev, S. V., Stanley, H. E. \& Havlin, S. Networks formed from interdependent networks. Nature Phys. 8, 40-48 (2012).

3. Son, S-W. et al. Percolation theory on interdependent networks based on epidemic spreading. Euro Phys Lett. 97, 16006 (2012).

4. Saumell-Mendiola, A., Serrano, M. Á. \& Boguñá, M. Epidemic spreading on interconnected networks. Phys. Rev. E 86, 026106 (2012).

5. Gómez, S. et al. Diffusion dynamics on multiplex networks. Phys. Rev. Lett. 110, 028701 (2013).

6. Aguirre, J., Papo, D. \& Buldú, J. M. Successful strategies for competing networks. Nature Phys. 9, 230-234 (2013).

7. Albert, R. \& Barabási, A-L. Statistical mechanics of complex networks. Rev. Mod. Phys. 74, 47-97 (2002).

8. Newman, M. E. J. Networks: An Introduction (Oxford Univ. Press, 2010).

9. Dorogovtsev, S. N., Goltsev, A. V. \& Mendes, J. F. F. Critical phenomena in complex networks. Rev. Mod. Phys. 80, 1275-1335 (2008).

10. Szella, M., Lambiotte, R. \& Thurner, S. Multirelational organization of large-scale social networks in an online world. Proc. Natl Acad. Sci. USA 107, 13636-13641 (2010)

11. Barthélemy, M. Spatial networks. Phys. Rep. 499, 1-101 (2011).

12. Albert, R., Jeong, H. \& Barabási, A-L. Error and attack tolerance of complex networks. Nature 406, 378-382 (2000).

13. Merris, R. Laplacian matrices of graphs: a survey. Linear Algebra Appl. 197-198, 143-176 (1994).

14. Chung, F., Lu, L. \& Vu, V. Spectra of random graphs with given expected degrees. Proc. Natl Acad. Sci. USA 100, 6313-6318 (2003).

15. Chung, F. Spectral Graph Theory (CBMS Regional Conference Series in Mathematics, American Mathematical Society, 1997).

16. Biyikoglu, T., Leydold, J. \& Stadler, P. F. Laplacian Eigenvectors of Graphs: Perron-Frobenius and Faber-Krahn Type Theorems (Lecture Notes in Mathematics, Springer, 2007).

17. Fielder, M. Algebraic connectivity of Graphs. Czech. Math. J. 23, 298-305 (1973).

18. Fielder, M. A property of eigenvectors of nonnegative symmetric matrices and its application to graph theory. Czech. Math. J. 25, 619-633 (1975).
19. Fielder, M. Laplacian of graphs and algebraic connectivity. Comb. Graph Theory 25, 57-70 (1989).

20. Mohar, B. Graph Theory, Combinatorics, and Applications 871-898 (Wiley, 1991).

21. Ng, A. Y., Jordan, M. I. \& Weiss, Y. Advances in Neural Information Processing Systems Vol. 14 (MIT Press, 2001).

22. Courant, R. Über die Eigenwerte bei den Differentialgleichungen der mathematischen Physik. Math. Z. 7, 1-57 (1920).

23. Fischer, E. Über quadratische Formen mit reellen Koeffizienten. Mon. Math. Phys. 16, 234-249 (1905).

24. Kolmogorov, V. \& Zabih, R. What energy functions can be minimized via graph cuts. IEEE Trans. Pattern Anal. 26, 65-81 (2004).

25. Reidys, C. M. \& Stadler, P. F. Combinatorial landscapes. SIAM Rev. 44, 3-54 (2002).

26. Mézard, M., Parisi, G. \& Virasoro, M. A. Spin Glass Theory and Beyond (World Scientific, 1987).

27. Grover, L. K. Local search and the local structure of NP-complete problems. Oper. Res. Lett. 12, 235-243 (1992).

28. Blundell, S. J. \& Blundell, K. M. Concepts in Thermal Physics (Oxford Univ. Press, 2008).

29. Jamakovic, A. \& Van Mieghem, P. in Proc. 7th Int. IFIP-TC6 Networking Conf. on AdHoc and Sensor Networks, Wireless Networks, Next Generation Internet 183-194 (Springer, 2008).

30. Parshani, R., Buldyrev, S. V. \& Havlin, S. Interdependent networks: Reducing the coupling strength leads to a change from a first to second order percolation transition. Phys. Rev. Lett. 105, 048701 (2010).

\section{Acknowledgements}

This work has been partially supported by the Spanish DGICYT Grants FIS2012-38266, FET projects PLEXMATH (318132) and the Generalitat de Catalunya 2009-SGR-838. F.R. acknowledges support from the Spanish Ministerio de Ciencia e Innovacion through the Ramón y Cajal programme. A.A. acknowledges the ICREA Academia and the James S. McDonnell Foundation.

\section{Author contributions}

F.R and A.A. designed and performed the research, and wrote the paper.

\section{Additional information}

Supplementary information is available in the online version of the paper. Reprints and permissions information is available online at www.nature.com/reprints.

Correspondence and requests for materials should be addressed to F.R.

\section{Competing financial interests}

The authors declare no competing financial interests. 